\title{
Natalidad y Mortalidad neonatal en el Hospital Universitario Central de Asturias durante los últimos diez años
}

\author{
María Díaz y Carmen Rodríguez \\ Hospital Universitario Central de Asturias (España)
}

\begin{abstract}
El estudio de los índices demográficos básicos de una comunidad es un factor muy importante para el conocimiento de la situación socioeconómica y sanitaria de dicha comunidad, así como para conocer su evolución demográfica. El objetivo es conocer las Tasas de Natalidad y Mortalidad Neonatal y Perinatal en el Hospital Universitario Central de Asturias en los últimos 10 años. En cuanto al método, el análisis descriptivo de los datos de nacimientos y fallecimientos (mortinatos y recién nacidos vivos muertos en el periodo neonatal), obtenidos del archivo de Memorias del Servicio de Neonatología del Hospital Universitario Centra de Asturias durante los últimos diez años, con el fin de estudiar y analizar las diferentes tasas de Mortalidad Neonatal y Perinatal. Los resultados son: en estos 10 años nacieron en el HUCA 24.674 recién nacidos totales (RNT), de los que 24.525 fueron recién nacidos vivos (RNV) y 149 mortinatos o recién nacidos muertos (RNM). Por edad gestacional, de los 24.525 RNV, 2.809 fueron menores de 37 semanas (11\%), 507 fueron menores de 32 semanas (2\%) y 192 fueron menores de 29 semanas de edad gestacional $(0,7 \%)$. De los $24.525 \mathrm{RNV}, 108$ fallecieron en los primeros 28 días de vida $(0,4 \%)$. La tasa de mortalidad neonatal total varió entre el 0,93 y el 6,98 por mil. Concluyendo, tras un aumento inicial de la natalidad en la última década, desde el año 2009 se ha objetivado un lento y constante descenso en el número de niños que nacen en el Hospital Universitario Central de Asturias. El porcentaje global de niños nacidos prematuros (menores de 37 semanas de edad gestacional) en este centro es del 11\%. Las tasas de mortalidad neonatal y perinatal son muy bajas, presentando una discreta variabilidad interanual.
\end{abstract}

Palabras clave: Recién nacido, natalidad, mortalidad perinatal, mortalidad neonatal.

Birthrate and neonatal mortality at the Asturias Central University Hospital during the last ten years. The study of the basic demographic indexes of a community is a very important factor for the knowledge of the socioeconomic and health situation of said community, as well as to know its demographic evolution. The objective is to know the Birth and Neonatal and Perinatal Mortality Rates at the Asturias Central University Hospital in the last 10 years. The method is descriptive analysis of birth and death data (stillbirths and live newborns killed in the neonatal period), obtained from the archive of Memories of the Neonatology Service of the Asturias Central University Hospital during the last ten years, in order to study and analyze the different rates of Neonatal and Perinatal Mortality. The results are in these 10 years 24,674 total newborns (RNT) were born in HUCA, of which 24,525 were live newborns (RNV) and 149 stillbirths or dead newborns (RNM). By gestational age, of the 24,525 RNV, 2,809 were younger than 37 weeks (11\%), 507 were younger than 32 weeks $(2 \%)$ and 192 were younger than 29 weeks of gestational age $(0.7 \%)$. Of the $24,525 \mathrm{RNV}, 108$ died in the first 28 days of life $(0.4 \%)$. The total neonatal mortality rate varied between 0.93 and 6.98 per thousand. The conclusions are after an initial increase in birth rates in the last decade, since 2009 there has been a slow and steady decrease in the number of children born in the Asturias Central University Hospital. The overall percentage of children born prematurely (under 37 weeks of gestational age) in this center is $11 \%$. The rates of neonatal and perinatal mortality are very low, presenting a discrete interannual variability.

Keywords: Newborn, birth, perinatal mortality, neonatal mortality.

Correspondencia: María Díaz Gómez. Hospital Universitario Central de Asturias. Avenida de Roma, s/n. 33011. Oviedo (España).maria.diazgomez@ hotmail.com 
El estudio de los índices demográficos básicos de una comunidad es un factor muy importante para el conocimiento de la situación socioeconómica y sanitaria de dicha comunidad, así como para conocer su evolución demográfica. Nuestra sociedad ha cambiado de forma muy llamativa en los últimos decenios. De una sociedad en desarrollo, con altas tasas de natalidad y una pirámide poblacional de base ancha, hemos pasado a una sociedad muy desarrollada, donde la natalidad es muy baja y la pirámide poblacional está invertida. Esto hace peligrar el sistema de pensiones públicos en España y el estado de bienestar de todos los españoles.

Si analizamos el número de nacimientos durante el primer periodo de cada año, según datos del INE en 2018, el descenso de nacimientos fue evidente en todas las comunidades autónomas, siendo la Rioja con un descenso del 13,7\% seguido de Extremadura con un descenso del 10,3\% donde el descenso fue más acusado. Por su parte el Instituto Nacional de Estadística destaca un aumento de nacimientos en madres extranjeras, lo que supone 2 de cada 10 niños nacidos.

El deterioro demográfico de nuestro país comenzó con la crisis económica del año 2008 que hizo que gran parte de los inmigrantes regresasen a sus países de origen buscando nuevas oportunidades de futuro.

En los últimos años, Asturias ha presentado unas cifras de defunciones en constante aumento y unas cifras de nacimiento en constante descenso. Así, en el primer semestre de 2015 se registraron en nuestra Comunidad Autónoma un total de 7.368 defunciones, lo que supone más del doble que los 3.235 nacimientos contabilizados en ese mismo periodo. Es decir, hubo 4.133 defunciones más que nacimientos (INE, 2014).

Nuestra comunidad autónoma ofrece la menor tasa de fecundidad de todo el país, con una media de 1, 03 hijos por mujer, situado por debajo de la media de 1,31 y muy lejos de otras regiones como Ceuta con una media de 1,81 hijos por mujer.

Si comparamos los datos de nacimientos y de muertes en la región (según cifras del Instituto Nacional de Estadística) hay un saldo vegetativo de 7140 personas. Pero no solo nos encontramos en la cola a nivel nacional, las estadísticas europeas colocan a Asturias entre las regiones con la tasa de natalidad más baja, siendo en el año 2014 el área que menos nacimientos registró de toda Europa.

La conclusión es que el crecimiento vegetativo, diferencia entre nacimientos y fallecimientos, cae vertiginosamente en nuestra población. Así lo demuestran los datos del Instituto Nacional de Estadística (INE) (1).

La serie histórica de mortalidad en nuestra región se puede ver en la tabla 1.

Tabla 1. Número de fallecimientos en Asturias en los últimos años (SADEI, 2014)

\begin{tabular}{lc}
\hline Año & $N^{\circ}$ Defunciones \\
\hline 2000 & 12393 \\
\hline 2005 & 12703 \\
\hline 2010 & 12725 \\
\hline 2014 & 12813 \\
\hline Datos: INE (2014).
\end{tabular}


Con estas cifras, Asturias vuelve a encabezar un año más la tasa bruta de mortalidad en España. Sin duda, estos datos son muy preocupantes y deberían llevar a nuestras instituciones a estudiar las causas que condujeron a nuestra Comunidad a ocupar ese ranking durante los últimos años y extraer las conclusiones oportunas.

Con estas cifras, Asturias vuelve a encabezar un año más la tasa bruta de mortalidad en España. Sin duda, estos datos son muy preocupantes y deberían llevar a nuestras instituciones a estudiar las causas que condujeron a nuestra Comunidad a ocupar ese ranking durante los últimos años y extraer las conclusiones oportunas.

Combinando las tasas de natalidad y mortalidad podemos comprobar como en estos momentos nuestra tasa de mortalidad casi duplica nuestra tasa de natalidad, con una tasa de crecimiento vegetativo del $-5 \%$ (tabla 2 ).

Tabla 2. Indicadores demográficos del 2014 para Asturias (INE, 2014)

\begin{tabular}{lcc}
\hline & 2013 & 2014 \\
\hline Nacimientos & 6.671 & 6.600 \\
\hline Tasa bruta de Natalidad (por mil) & 6,28 & 6,26 \\
\hline Defunciones & 12.722 & 12.813 \\
\hline Tasa bruta de mortalidad (por mil) & 11,97 & 12,16 \\
\hline Saldo vegetativo & -6.051 & -6.213 \\
\hline Tasa de crecimiento vegetativo (por cien) & $-5,69$ & $-5,89$ \\
\hline Datos: INE (2014). & &
\end{tabular}

Con estos datos a la vista, la tendencia en el Principado de Asturias durante estos años ha sido de una caída alarmante de los índices de natalidad y un aumento de los índices de mortalidad, lo cual está indicando un envejecimiento de la población asturiana. Asturias se está convirtiendo en una Comunidad con una población vieja.

La inmigración, a pesar de su descenso ha sido pieza clave durante este último siglo para el crecimiento poblacional. En el año 2014 de los 6.600 nacidos en Asturias, 561 eran nacidos de madre extranjera. En la tabla 3 podemos ver los datos de los años 2010 a 2014 (INE, 2014).

Tabla 3. Nacimientos en Asturias de madres extranjeras (INE, 2014)

\begin{tabular}{lccccc}
\hline & 2010 & 2011 & 2012 & 2013 & 2014 \\
\hline Total & 794 & 751 & 716 & 638 & 561 \\
\hline$\%$ sobre el total de nacimientos & 10,23 & 9,65 & 9,39 & 9,56 & 8,50 \\
\hline Datos: INE (2014).
\end{tabular}

Todos estos datos, y otros ya publicados por el INE, nos indican que el tramo de población con mayor porcentaje ha pasado de la comprendida entre las edades de 25 a 29 años en 2001, a ser de 35 a 39 años en 2014. Como consecuencia de estos desajustes poblacionales, por otra parte, se producen desproporciones en cuanto a la población activa, disminución de ingresos en la caja de la Seguridad Social, riesgo de quiebra en el estado del bienestar y aumento del gasto sanitario (Angulo, 2014). 
La Natalidad hace referencia a la proporción de nacimientos ocurridos en una población dada y en un periodo de tiempo determinado.

Demográficamente la Tasa de Natalidad determina el nivel de fecundidad de un país junto con la Tasa de Fecundidad General y la Tasa Global de Fecundidad, que puede definirse como la medida entre los nacimientos ocurridos en un determinado periodo de tiempo por cada mil habitantes en relación a la población total (Calleja et al., 2006).

El crecimiento de la población por el número de nacimientos se denomina crecimiento vegetativo y se calcula mediante la diferencia entre nacimientos y muertes en un periodo de tiempo y en un lugar determinado. Hay una serie de indicadores básicos que determinan la natalidad de una región como son la edad de la madre, el nivel de estudios o su situación conyugal, la atención sanitaria el peso del recién nacido al nacer o su edad gestacional.

Es un hecho que durante el último siglo la natalidad ha ido disminuyendo de manera evidente en nuestro país. Aunque esta disminución no ha ocurrido de la misma manera en todo el territorio español. La mayor parte de nuestra población la componen personas mayores de 65 años, descendiendo así las tasas de mortalidad y de natalidad. Entre los factores atribuibles al descenso de la natalidad en España encontramos la planificación familiar (límite en el número de hijos, elección del momento adecuado) edad de los padres, factores económicos, acceso a métodos anticonceptivos fiables, cambio de valores, etc. La disminución de la natalidad es un fenómeno que se viene dando en toda Europa Occidental desde hace unas décadas. Las causas de este fenómeno son complejas y diversas. Tras los años del baby-boom, España sufrió un descenso de los nacimientos, lento pero progresivo, hasta llegar a un mínimo alrededor del año 1995-96. A partir de ese año, y como consecuencia de los años de crecimiento económico, la natalidad ascendió también paulatinamente hasta el final de la primera década del siglo XXI (2009-2010), para posteriormente volver a bajar (De Llano et al., 2006; INE, 2014).

Este mismo fenómeno demográfico ocurrió en Asturias, aunque con tasas de fecundidad y natalidad todavía más bajas, lo que sin duda representa un gran problema demográfico.

La crisis económica, los cambios sociales, culturales y religiosos, el final de la edad fértil de las mujeres nacidas en los años del baby-boom y la escasa atracción que tiene Asturias para la llegada de inmigrantes son las razones por las que nuestra región presenta en la actualidad las tasas más bajas de natalidad de toda España.

Entendemos por mortalidad del recién nacido o mortalidad neonatal aquella que hace referencia al número de fallecidos entre los 28 días y el primer año de vida (SENEO, 2014). Así, la Tasa de Mortalidad nos indica el número de recién nacidos fallecidos por cada mil recién nacidos a lo largo de un año.

Según la duración del embarazo y el momento del fallecimiento podemos 
distinguir entre mortalidad fetal y mortalidad Neonatal.

Dentro de la mortalidad fetal, diferenciamos entre:

-Mortalidad fetal temprana, la que se produce entre la 20 y la 27 semana de gestación.

-Mortalidad fetal tardía, la ocurrida después de la 28 semana de gestación.

Por su parte, la mortalidad neonatal se puede dividir en:

-Mortalidad neonatal temprana, dentro de los 7 primeros días de vida.

-Mortalidad neonatal tardía, entre el 8 y el 28 día de vida.

Entendemos como mortalidad perinatal la que agrupa la muerte fetal tardía y la muerte neonatal temprana, comprendiendo entre la 28 semana de gestación hasta el 7 día de vida.

Entre las causas más importantes de muerte neonatal podemos encontrar (Cruz, Lapresta, José, Andrés, y Villacampa, 2004; Tosca, Aguilera, y Bellido, 2002):

-La prematuridad, pero sobre todo la prematuridad extrema.

-El nacimiento con bajo peso, sobre todo el peso extremadamente bajo.

-Las infecciones neonatales (verticales y nosocomiales).

-Las situaciones de depresión perinatal grave (o síndromes hipóxico- isquémicos).

-Las anomalías congénitas.

-Los problemas de la gestación en relación a enfermedades maternas (preeclampsia, diabetes...) y a problemas de la placenta (desprendimientos).

-Los nacimientos prematuros junto a los nacimientos de bajo peso son responsables del $25 \%$ de las muertes neonatales, por su parte los defectos al nacimiento producen el $20 \%$ de las muertes en edad neonatal.

La mortalidad neonatal es un indicador de la calidad asistencial básica de un país y se utiliza como dato o estadística para calcular la calidad de vida de los niños en una determinada región, así como para determinar el nivel socioeconómico. Por ello, resultaría útil establecer análisis periódicos de los mismos a fin de analizar cómo evolucionan en el tiempo dichos indicadores (Sopena, Guillen, Marques, y Cunat, 2012). Esta tasa se ve influida por cuestiones como el acceso a la salud, la alimentación o el medio ambiente sano. Si el índice de mortalidad infantil es alto, estaríamos ante una región con un alto riesgo social y demográfico puesto que no se asegura una adecuada calidad de vida en su población, disminuyendo por tanto la esperanza de vida de la misma.

Actualmente todas las tasas de mortalidad infantil en España están sufriendo una progresiva reducción en los últimos años para todas las edades y en cualquier circunstancia. Todo ello, a consecuencia del desarrollo económico y social, los grandes avances médicos y la mejoría en la asistencia sanitaria de nuestro país (INE, 2014).

Muy notable es el descenso de la mortalidad neonatal y el aumento de la supervivencia en los niños prematuros y de muy bajo peso. 
Las mejoras en salud han sido evidentes en algunos campos como la disminución de la mortalidad relacionada con anomalías genéticas, mejora de la supervivencia de niños con discapacidades y enfermedades crónicas, disminución de las infecciones por VIH o la mejora en los programas de educación sanitaria. Sin embargo, otros problemas sociales que nos atañen requieren de una mayor atención e implicación como son el aumento de las patologías relacionadas con la calidad ambiental, el aumento de los trastornos neuropsíquicos, las patologías relacionadas con la alimentación o el estilo de vida (tabaco, alcohol...).

El objetivo de este estudio es conocer las cifras de natalidad y mortalidad perinatal del Hospital Universitario Central de Asturias en los últimos 10 años.

En concreto:

-Conocer los datos generales de natalidad.

-Conocer el porcentaje de nacimientos de niños prematuros.

-Conocer el porcentaje de recién nacidos según peso.

-Conocer los datos de mortalidad neonatal y perinatal total, así como sus respectivas subclases.

\section{MÉTODO}

Análisis descriptivo de los datos de nacimientos y fallecimientos (mortinatos y recién nacidos vivos muertos en el periodo neonatal), obtenidos del archivo de Memorias del Servicio de Neonatología del Hospital Universitario Central de Asturias durante los últimos diez años, con el fin de estudiar y analizar las diferentes tasas de Mortalidad Neonatal y Perinatal.

\section{Mortalidad fetal}

Cociente resultante de dividir el número de mortinatos (o fetos mayores de 22 semanas de EG) nacidos muertos entre el número total de recién nacidos en el periodo de estudio.

\section{Mortalidad perinatal precoz}

Cociente resultante de dividir el número de mortinatos (o fetos mayores de 22 semanas de EG) nacidos muertos, más los recién nacidos vivos fallecidos durante los primeros 7 días de vida, entre el número total de recién nacidos en el periodo de estudio.

\section{Mortalidad perinatal total}

Cociente resultante de dividir el número de mortinatos (o fetos mayores de 22 semanas de EG) nacidos muertos, más los recién nacidos vivos fallecidos durante los primeros 28 días de vida, entre el número total de recién nacidos en el periodo de estudio. 


\section{Mortalidad neonatal inmediata (0-24 horas)}

Cociente resultante de dividir el número de recién nacidos vivos fallecidos en las primeras 24 horas de vida, entre el número total de recién nacidos vivos.

\section{Mortalidad neonatal precoz (0-7 días)}

Cociente resultante de dividir el número de recién nacidos vivos fallecidos en los primeros7 días de vida, entre el número total de recién nacidos vivos.

\section{Mortalidad neonatal tardía (8-28 días)}

Cociente resultante de dividir el número de recién nacidos vivos fallecidos entre el 8 y el 28 día de vida, entre el número total de recién nacidos vivos.

\section{Mortalidad neonatal total (0-28 días)}

Cociente resultante de dividir el número de recién nacidos vivos fallecidos desde el nacimiento y el 28 día de vida, entre el número total de recién nacidos vivos.

Todos estos cocientes se expresan por cada 1000 (cada $1000 \mathrm{RN}$ totales o cada $1000 \mathrm{RN}$ vivos).

\section{RESULTADOS}

Entre el 1-1-2006 y el 31-12-2015 hubo un total de 24.032 partos en el HUCA. De ellos, 23.400 fueron partos sencillos, 620 partos gemelares y 12 partos triples.

\section{Natalidad}

En estos 10 años, por lo tanto, nacieron en el HUCA 24.674 recién nacidos totales (RNT), de los que 24.525 fueron recién nacidos vivos (RNV) y 149 mortinatos o recién nacidos muertos.

En la tabla 4 se pueden leer los datos de natalidad en estos 10 años.

Tabla 4. Natalidad 2006-2015 en el HUCA

\begin{tabular}{|c|c|c|c|c|c|c|c|c|c|c|}
\hline & 2006 & 2007 & 2008 & 2009 & 2010 & 2011 & 2012 & 2013 & 2014 & 2015 \\
\hline $\mathrm{N}^{\circ}$ partos & 2463 & 2467 & 2632 & 2707 & 2514 & 2523 & 2377 & 2172 & 2059 & 2118 \\
\hline Tipo partos: & 2409 & 2412 & 2577 & 2630 & 2462 & 2449 & 2283 & 2109 & 2003 & 2066 \\
\hline $\begin{array}{l}\text { Gemelare } \\
\text { s Triples }\end{array}$ & 1 & 1 & 2 & 3 & 1 & 0 & 4 & 0 & 0 & 0 \\
\hline $\mathrm{N}^{\circ} \mathrm{RN}$ totales & 2518 & 2523 & 2689 & 2787 & 2565 & 2597 & 2475 & 2235 & 2115 & 2170 \\
\hline RN vivos totales & 2506 & 2507 & 2670 & 2776 & 2549 & 2577 & 2461 & 2220 & 2100 & 2159 \\
\hline RNV menores de $1500 \mathrm{~g}$ & 48 & 47 & 57 & 67 & 50 & 53 & 46 & 37 & 37 & 37 \\
\hline RNV menores de $1000 \mathrm{~g}$ & 17 & 17 & 26 & 25 & 16 & 28 & 14 & 11 & 11 & 10 \\
\hline RNV menores 37 sem & 299 & 312 & 357 & 335 & 335 & 275 & 249 & 229 & 227 & 191 \\
\hline RNV menores 32 sem & 53 & 69 & 65 & 58 & 58 & 57 & 46 & 36 & 36 & 29 \\
\hline RNV menores 29 sem & 19 & 18 & 28 & 22 & 28 & 28 & 20 & 11 & 12 & 6 \\
\hline
\end{tabular}


En las figuras 2, 3 y 4 se puede ver la evolución de estas cifras por año (figura 1 evolución de los recién nacidos totales, figura 2 evolución de los recién nacidos por peso y la figura 3 evolución de los recién nacidos por edad gestacional).

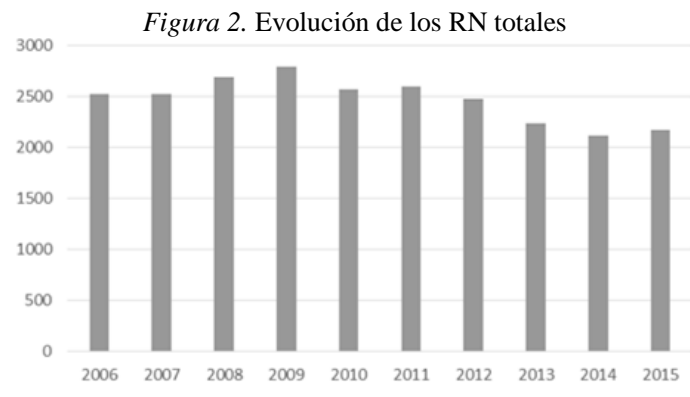

Figura 3. Evolución de los RN por pesos

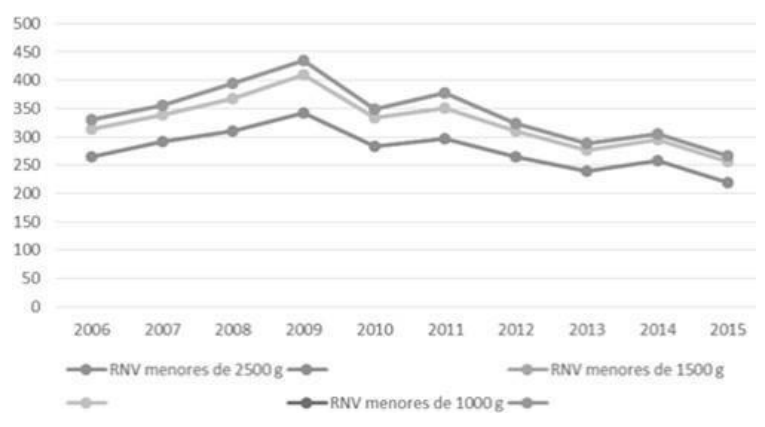

Figura 4. Evolución de los RN por edad gestacional

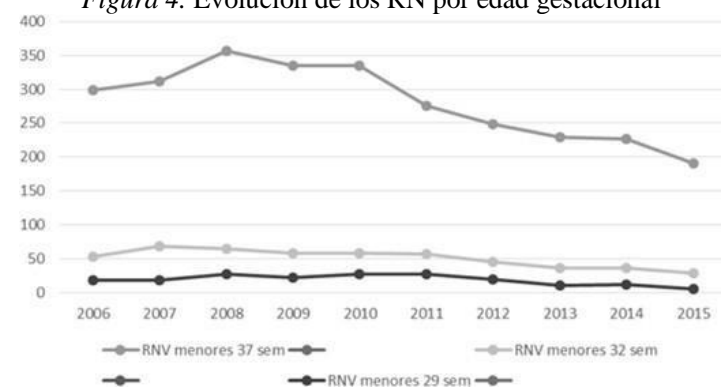

Mortalidad perinatal

De los 24.674 RNT que nacieron en el HUCA en estos 10 años, 166 fueron neonatos muertos o mortinatos $(0,6 \%)$. 
En la Figura 5 se puede ver la evolución del número de los RN muertos o mortinatos por año y en la Figura 6 se puede ver la evolución de los RNV fallecidos en los primeros 28 días de vida en este decenio.
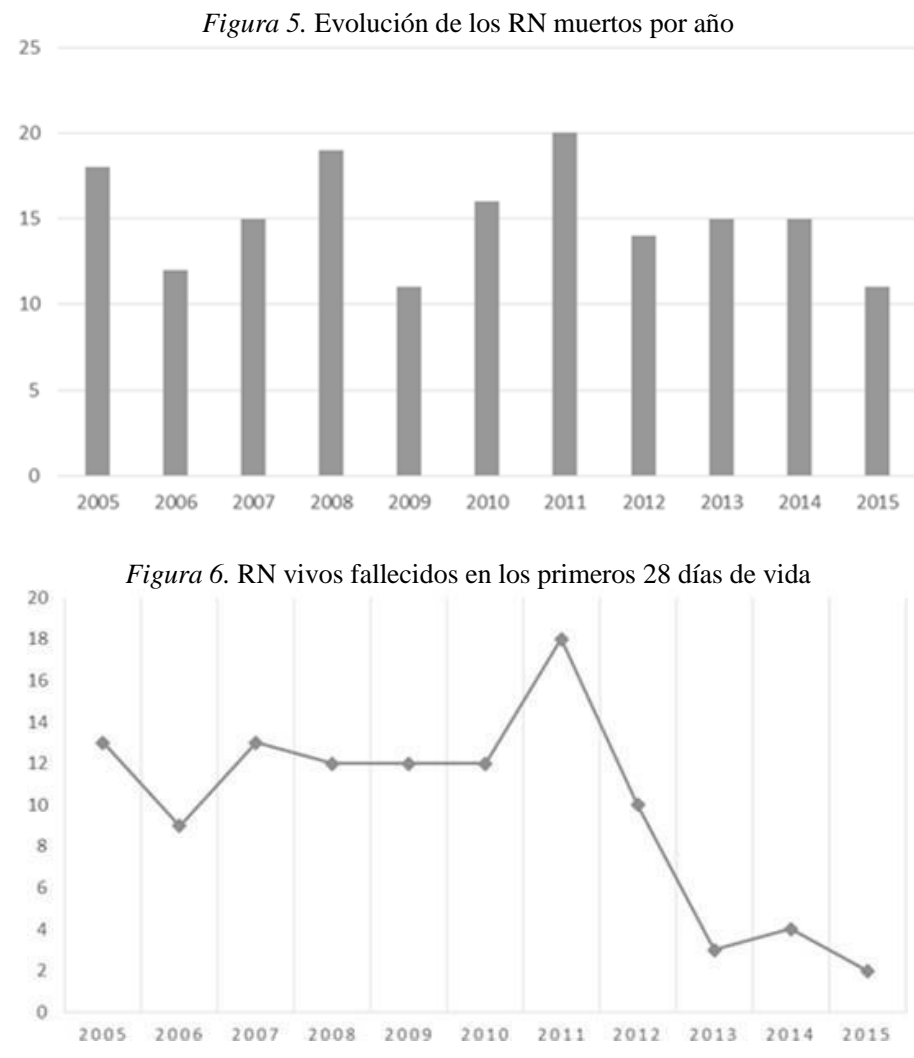

\section{Tasas de mortalidad}

En la Tabla V se pueden leer los datos de las distintas tasas de mortalidad perinatal en los últimos 10 años. MF: mortalidad fetal; MPP: mortalidad perinatal precoz; MPT: mortalidad perinatal total; MNT: mortalidad neonatal total; MNI: mortalidad neonatal inmediata; MNP: mortalidad neonatal precoz; MNT: mortalidad neonatal tardía. 
Tabla 5. Mortalidad en el HUCA en el decenio 2006-2015

\begin{tabular}{|c|c|c|c|c|c|c|c|c|c|c|c|}
\hline & 2005 & 2006 & 2007 & 2008 & 2009 & 2010 & 2011 & 2012 & 2013 & 2014 & 2015 \\
\hline $\mathrm{N}^{\circ} \mathrm{RN}$ totales & 2419 & 2518 & 2523 & 2689 & 2787 & 2565 & 2597 & 2475 & 2235 & 2115 & 2170 \\
\hline RN vivos totales & 2401 & 2506 & 2507 & 2670 & 2776 & 2549 & 2577 & 2461 & 2220 & 2100 & 2159 \\
\hline $\mathrm{RN}$ nacidos muertos & 18 & 12 & 15 & 19 & 11 & 16 & 20 & 14 & 15 & 15 & 11 \\
\hline $\begin{array}{l}\text { RNV de nuestro centro } \\
\text { fallecidos en periodo neonatal }\end{array}$ & 13 & 9 & 13 & 12 & 12 & 12 & 18 & 10 & 3 & 4 & 2 \\
\hline $\begin{array}{l}\text { RN vivos totales fallecidos en } \\
\text { nuestro Servicio }\end{array}$ & 17 & 12 & 22 & 21 & 13 & 16 & 19 & 17 & 5 & 7 & $5^{*}$ \\
\hline Mortalidad: & & & & & & & & & & & \\
\hline MF & 7,44 & 4,77 & 5,94 & 7,06 & 3,95 & 6,23 & 6,93 & 5,66 & 6,71 & 7,09 & 5,06 \\
\hline MPP & 11,16 & 7,94 & 8,72 & 10,4 & 6,46 & 9,75 & 9,63 & 9,29 & 8,05 & 8,04 & 5,06 \\
\hline MPT & 12,82 & 8,34 & 11,09 & 11,5 & 8,25 & 10,9 & 13,86 & 9,69 & 8,05 & 8,98 & 5,99 \\
\hline MNT & 5,41 & 3,59 & 5,18 & 4,49 & 4,32 & 4,7 & 6,98 & 4,06 & 1,35 & 1,90 & 0,93 \\
\hline MNI & 2,92 & 1,20 & 1,59 & 1,87 & 2,16 & 1,18 & 1,16 & 2,03 & 0,45 & 0,48 & 0 \\
\hline MNP & 3,75 & 3,19 & 2,79 & 3,37 & 2,52 & 3,53 & 2,72 & 3,65 & 1,35 & 0,95 & 0 \\
\hline MNTardía & 1,67 & 0,40 & 2,39 & 1,12 & 1,80 & 1,18 & 4,27 & 0,41 & 0 & 0,95 & 0,93 \\
\hline
\end{tabular}

\section{CONCLUSIONES/DISCUSIÓN}

Gracias a los datos obtenidos del archivo de Memorias del Servicio de Neonatología del HUCA durante los últimos diez años, se han podido estudiar y analizar los datos de Natalidad y Mortalidad Neonatal exhaustivamente, ya que la Memoria del Servicio cuenta con datos no sólo totales sino según los diferentes subtipos de mortalidad perinatal como son la fetal, neonatal precoz, neonatal tardía... que ayudan a establecer datos de mortalidad en las diferentes etapas.

Estas cifras exactas de nacimientos y fallecimientos en el periodo perinatal de los últimos diez años, muestran una tendencia clara de estabilidad o mejoría en las cifras de mortalidad y una tendencia al descenso en la natalidad desde el inicio de la crisis social y económica que atraviesa España en los últimos años.

Contrastando estos datos con otros estudios realizados previamente a nivel nacional, observamos cómo nuestra comunidad continúa encabezando la lista de la menor tasa de fecundidad del país con una media de 1,03 hijos por mujer.

Debemos tener en cuenta, que el Hospital Universitario Central de Asturias (HUCA), es el hospital de referencia para el resto de Asturias, lo que implica un aumento en las tasas de mortalidad ya que las gestaciones con problemas suelen derivarse al hospital de referencia, aumentando el número de casos de niños grandes prematuros o con problemas mal formativos con más probabilidad de morir.

Gijón por su parte, cuenta con un Hospital con una unidad de neonatología, por tanto, esta área sanitaria solo drena al HUCA los casos quirúrgicos, al contrario del resto de áreas sanitarias de la región que drenan a nuestro hospital todos los casos de patología neonatal. Comparando los datos obtenidos en nuestro estudio con los obtenidos en otros estudios españoles los resultados son muy similares, en todos ellos el descenso de la mortalidad es notable, así como el descenso marcado en la natalidad durante estos últimos años. 
Según muestra el INE en el año 2018 el descenso de nacimientos ha sido evidente en todas las comunidades autónomas, aumentando solo el número de nacimientos de madres extranjeras. Esto supone 2 de cada 10 niños nacidos en nuestro país.

Tras un aumento inicial de la natalidad en la última década, desde el año 2009 se ha objetivado un lento y constante descenso en el número de niños que nacen en el Hospital Universitario Central de Asturias.

El porcentaje global de niños nacidos prematuros (menores de 37 semanas de edad gestacional) en este centro es del $11 \%$.

Las tasas de mortalidad neonatal y perinatal son muy bajas, presentando una discreta variabilidad interanual.

\section{REFERENCIAS}

Angulo, J.V. (2004). Comportamiento de la natalidad en España y perspectivas de futuro sobre el empleo, el sistema educativo y el diálogo social. En J.M. Díaz y J.J. Jaraíz (Eds.), Familia, educación y empleo: hacia una concepción integral e integradora (pp. 145-160). Civitas.

Calleja, J.R. Martínez, M.G. Rodríguez, R.R. Pereira, M.P. Villaverde, C.M., y Álvarez, I.F. (2006). Evolución de la natalidad y mortalidad neonatal y perinatal en el Área de Salud El Bierzo: 1992-2004. Boletín de Pediatría, 46, 39-45.

Cruz, E., Lapresta, M., José, Y., Andrés, P., y Villacampa, A. (2004). Mortalidad perinatal. Distribución según la edad gestacional y el peso al nacimiento. Causas más frecuentes. Revisión epidemiológica de 10 años. Clínica e Investigación en Ginecología y Obstetricia, 31(9), 323-327.

De Llano, J.A., López, S.A., Leiza, J.G., Rubio, C.Q., Candela, R.C., y Ramalle-Gómara, E. (2015). Evolución de la natalidad en España. Análisis de la tendencia de los nacimientos entre 1941 y 2010. Anales de Pediatría, 82(1), 1-e6.

Instituto Nacional de Estadística (2014). Indicadores demográficos del 2014 para Asturias. Recuperado de: http://www.ine.es.

Instituto Nacional de Estadística (2014). Nacimientos en Asturias de madres extranjeras. Recuperado en: http://www.ine.es.

Sociedad Asturiana de Estudios Económicos e Industriales (2014). Datos básicos de Asturias 2014.

Sopena, F.M., Guillen, A.M., Marqués, A.G., y Cunat, V.A. (2012). Análisis de la mortalidad neonatal en el Hospital Universitario La Fe de Valencia. Años 1971- 2009. Anales de Pediatría, 77(5), 309-316.

Tosca, R., Aguilera, R., y Bellido, J. (2002). Causas de mortalidad neonatal en la Comunidad Valenciana. Anales de Pediatría, 57(6), 565-569

Recibido: 06 de septiembre de 2019 Recepción Modificaciones: 01 de octubre de 2019 Aceptado: 02 de octubre de 2019 